\title{
DISEÑO DE ESQUEMAS DE MODULACIÓN PARA UN SISTEMA DE POSICIONAMIENTO LOCAL ULTRASÓNICO CODIFICADO CON SECUENCIAS ZADOFF-CHU
}

\author{
S. Murano ${ }^{1,2}$, M. C. Pérez ${ }^{1}$, C. J. Bleakley ${ }^{3}$, J. Ureña ${ }^{1}$, C. De Marziani ${ }^{2}$ \\ santiago.murano@edu.uah.es, mcarmen.perezr@uah.es \\ ${ }^{1}$ Departamento de Electrónica, Universidad de Alcalá, España \\ ${ }^{2}$ Departamento de Electrónica, Universidad Nacional de la Patagonia San Juan Bosco, Argentina \\ ${ }^{3}$ School of Computer Science, University College Dublin, Ireland
}

\begin{abstract}
Resumen
Muchos sistemas de posicionamiento local ultrasónicos (U-LPS), consisten en varios transductores o balizas fijas emitiendo simultáneamente señales acústicas ultrasónicas, cuyo tiempo de llegada o diferencia de tiempos de llegada es estimado en un receptor móvil. El empleo de técnicas de codificación permite que el receptor sea capaz de separar cada una de ellas, identificar los transmisores y mitigar los efectos adversos que afecten a la señal, tales como el multicamino, el efecto cerca-lejos, la interferencia de acceso múltiple o el desplazamiento Doppler. El diseño de la forma de onda (codificación y modulación) desempeña un papel fundamental en la determinación del rendimiento de dicho sistema. En este trabajo se compara el rendimiento de tres técnicas de modulación (QPSK, OFDM y FHSS) para la transmisión de secuencias Zadoff-Chu (ZC) en un U-LPS. Las simulaciones realizadas indican que, en detección no coherente, la modulación QPSK de secuencias ZC ofrece mayor precisión en condiciones realistas que los otros esquemas de modulación.
\end{abstract}

Palabras clave: Codificación Zadoff-Chu, Frequency Hopping, OFDM, QPSK, ultrasonidos, posicionamiento local

\section{INTRODUCCIÓN}

Los sistemas de posicionamiento en interiores son una tecnología importante para muchas aplicaciones, incluyendo la navegación personal o robótica, seguridad de los dispositivos, publicidad basada en la ubicación, entre otras. La emisión de señales ultrasónicas permite obtener un posicionamiento de alta precisión (centimétrico), gracias a la baja velocidad de propagación del sonido en el aire. Para lograr una alta precisión, un sistema de posicionamiento local ultrasónico (U-LPS) requiere medidas de tiempo: tiempo de llegada (ToA, $t i$ me of arrival) o diferencias de tiempo de llegada (DToA, Differences of time of arrival) en caso de ausencia de sincronismo entre las balizas emisoras y el receptor. En ambos casos, el ToA de la señal ultrasónica debe estimarse con alta precisión, por lo que la señal utilizada debe ser lo suficientemente robusta frente a efectos adversos, incluyendo: multicamino, multi-emisión, efecto cerca-lejos y el desplazamiento Doppler que surge del movimiento del receptor. Además, la señal suele estar limitada por el ancho de banda disponible en el canal, y más específicamente por los transductores empleados.

La codificación de la señal a emitir permite mejorar el desempeño del sistema y mitigar los efectos comentados anteriormente. Como alternativa a códigos pseudo-aleatorios tradicionales [7, 8], las secuencias Zadoff-Chu (ZC) proporcionan un buen rendimiento en sistemas de medida de distancia con ultrasonido, sobretodo si se encuentran afectados por el efecto Doppler [5]. Las secuencias ZC son polifásicas de valores complejos, con amplitud unitaria constante, y auto-correlación (AC) cero para desplazamientos no nulos. Actualmente son utilizadas en el estándar de comunicaciones Long Term Evolution (LTE) [1].

En general, los sistemas ultrasónicos basados en técnicas de codificación o compresión de pulsos, requieren la modulación de la secuencia codificada para adaptarla al ancho de banda de los transductores empleados. Esta modulación, y posterior demodulación (generalmente no coherente en sistemas U-LPS) implica una degradación de las propiedades de correlación de los códigos. Este trabajo compara el rendimiento de tres esquemas de modulación para la transmisión de códigos ZC dentro del ancho de banda disponible de un transductor ultrasónico típico. Los tres esquemas a comparar son: modulación en fase cuaternaria (QPSK, quaternary phase shift keying), espectro ensanchado por salto de frecuencia (FHSS, frequency hopping spread spectrum) y por división de frecuencias ortogonales (OFDM, orthogonal frequency division multiplexing). El rendimiento de estos esquemas se compara considerando condiciones típicas de degradación de la señal ultrasónica en interiores con múltiples emisores. 
El resto del documento está estructurado de la siguiente manera. La siguiente sección describe el diseño de las señales a emitir. La sección tres describe el procedimiento de evaluación y presenta los resultados de la simulación. Los hallazgos se discuten en la sección cuatro y la sección cinco concluye el documento.

\section{DISEÑO DE LAS SEÑALES}

\subsection{SECUENCIAS ZADOFF-CHU}

Las secuencias Zadoff-Chu, son códigos polifásicos y complejos de valor unitario. Se encuentran en el grupo de secuencias CAZAC (constant amplitude zero auto-correlation) debido a sus propiedades periódicas de correlación cero para desplazamientos no nulos. Una secuencia ZC $Z_{r_{k}}$ de longitud $L_{Z C}$, puede definirse como:

$$
Z_{r_{k}}[l]=e^{i \theta_{l}}, \quad l=0,1, \ldots L_{Z C}-1
$$

donde $\theta_{l}$ está dada por:

$$
\theta_{l}= \begin{cases}\frac{\pi r_{k} l^{2}}{L_{Z C}}, & \text { si } L_{Z C} \text { es par } \\ \frac{\pi r_{k} l(l+1)}{L_{Z C}}, & \text { si } L_{Z C} \text { es impar }\end{cases}
$$

La raiz de generación $r_{k}=1,2, \ldots, L_{Z C}-1$ es un número entero y primo relativo a $L_{Z C}[3]$, que permite obtener secuencias con baja correlación cruzada (CC).

En [2], se demostró que la función de autocorrelación (AC) periódica para una secuencia ZC satisface (3), para cualquier desplazamiento $\tau$. Esto mismo se puede verificar en la figura 1.

$$
R_{Z_{r_{k}}}[\tau]=\left\{\begin{array}{llll}
L_{Z C}, & \tau & \bmod & L_{Z C}=0 \\
0, & \tau & \bmod & L_{Z C} \neq 0
\end{array}\right.
$$

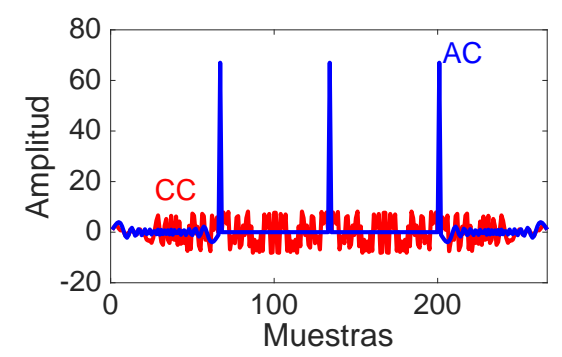

Figura 1: Función de autocorrelación y correlación cruzada para una emisión periódica de 3 secuencias ZC.

\subsection{MODULACIÓN QPSK}

Las secuencias ZC pueden ser moduladas en fase, utilizando para la parte real una onda cosenoi- dal y para la parte imaginaria una senoidal con la misma frecuencia de portadora. La diferencia de ambas señales es enviada al $k$-ésimo transductor como se indica en (4):

$$
\begin{aligned}
C[n]= & \cos \left(2 \pi f_{c} n\right)-i \cdot \sin \left(2 \pi f_{c} n\right) \\
& 0 \leq n / f_{s} \leq O_{f} N_{c} / f_{s} \\
x_{Q P S K_{k}}(n)= & \sum_{l=0}^{L_{Z C}-1}\left[\operatorname{Re}\left(Z_{r_{k}}(l)\right) \cdot \operatorname{Re}(C[n])\right. \\
& \left.-\operatorname{Im}\left(Z_{r_{k}}(l)\right) \cdot \operatorname{Im}(C[n])\right]
\end{aligned}
$$

donde $C[n]$ es la portadora compleja centrada a una frecuencia $f_{c}$, con una duración de símbolo que depende del factor de sobremuestreo $O_{f}=$ $f_{s} / f_{c}$, siendo $f_{s}$ la frecuencia de muestreo, y del número de ciclos por símbolo $N_{c}$.

La figura 2 muestra el procesamiento de la señal en las etapas de emisión y recepción para la estimación del ToA. Nótese que en el receptor, la correlación se realiza directamente con la señal emitida $x_{Q P S K_{k}}(n)$ para simplificar el proceso de detección.

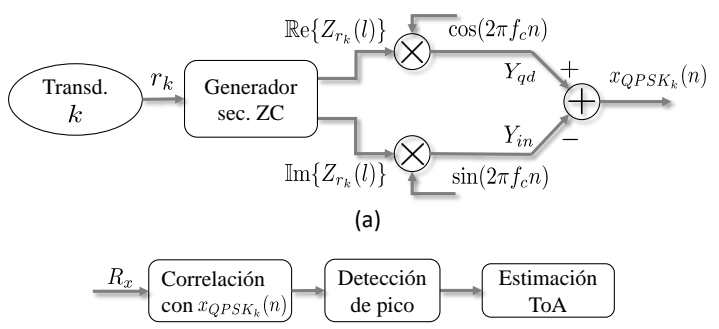

(b)

Figura 2: Esquema del emisor (a) y receptor (b) QPSK empleando secuencias ZC para el $k$-ésimo transductor.

\subsection{MODULACIÓN POR SALTO DE FRECUENCIA}

En la modulación FHSS, la señal a transmitir es propagada utilizando una portadora que alterna o "salta" de un canal a otro siguiendo un patrón, de modo que la señal permanece en el canal durante breves períodos de tiempo. En este trabajo se consideraron dos formas de salto de frecuencia: SlowFH y Fast-FH [6]. En Slow-FH, se transmite toda la secuencia ZC antes de cambiar de portadora. En contraste, Fast-FH salta para cada elemento de la secuencia. La figura 3 muestra el espectrograma de ambas señales para un único emisor.

Las secuencias ZC son moduladas utilizando QPSK, cambiando las frecuencias de portadora de acuerdo al patrón de saltos. La portadora también es multiplicada por una ventana Hanning, para 


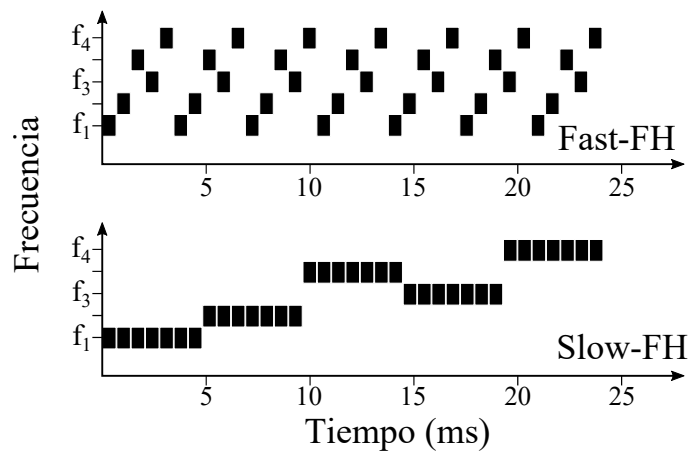

Figura 3: Espectrograma para FastFH (arriba) y SlowFH (abajo) para un único emisor codificado con la secuencia de saltos $\left\{f_{1}, f_{2}, f_{4}, f_{3}, f_{5}\right\}$.

reducir la amplitud de la señal en los cambios de fase, y suavizar el espectro en frecuencia. Esta modulación se puede describir según (5):

$$
\begin{aligned}
\operatorname{Hann}(t)= & \frac{1}{2}\left[1-\cos \left(\frac{2 \pi t}{T_{b}}\right)\right] \\
C_{k}(t)= & {\left[\cos \left(2 \pi f_{k} t+\phi\right)\right.} \\
& \left.-i \cdot \operatorname{sen}\left(2 \pi f_{k} t+\phi\right)\right] \cdot \operatorname{Hann}(t) \\
x_{F H_{k}}(t)= & \sum_{l=0}^{L_{Z C}-1}\left[\operatorname{Re}\left(Z_{r_{k}}(l)\right) \cdot \operatorname{Re}\left(C_{k}(t)\right)\right. \\
- & \left.\mathbb{I m}\left(Z_{r_{k}}(l)\right) \cdot \operatorname{Im}\left(C_{k}(t)\right)\right] \cdot \Pi_{T_{b}}(t)
\end{aligned}
$$

donde $T_{b}$ es la duración del símbolo, cuya duración mínima depende del ancho de banda disponible $B W=\frac{2}{T_{b}}[1] ; Z_{r_{k}}$ es la secuencia ZC generada con la raíz $r_{k}$ de la $k$-ésima baliza; el pulso rectangular $\Pi_{T_{b}}$ es igual a uno para $0 \leq t \leq T_{b}$ y cero en cualquier otro caso; $C_{k}(t)$ es la portadora multiplicada por la ventana Hanning, y $f_{k}$ es el conjunto de frecuencias sobre las cuales se realizan los saltos. En Slow-FH, $f_{k}$ es cambiada cada $L_{Z C}$ muestras, mientras que en Fast-FH, se hace para cada bit de la secuencia. La figura 4 muestra el patrón de transmisión por cada baliza o transductor ultrasónico (se han considerado cinco: B1-B5).

La figura 5(a) muestra el esquema de transmisión para ambas alternativas. Como se mencionó anteriormente, cada transductor tiene una secuencia ZC única, que en conjunto con los saltos de frecuencia aseguran la ortogonalidad y evitan, o mitigan, la colisión entre señales. En el receptor, al igual que en el esquema anterior, la correlación se realiza directamente con la señal emitida modulada $x_{F H_{k}}(t)$.

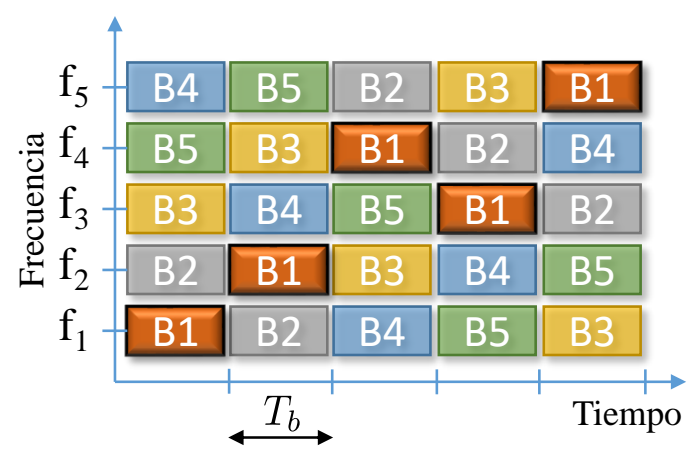

Figura 4: Patrón de emisión para cinco balizas diferentes.

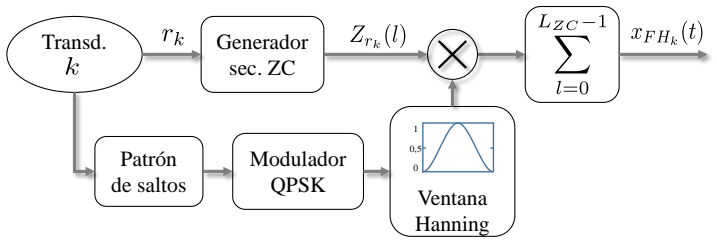

(a)

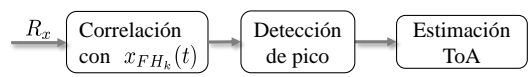

(b)

Figura 5: Esquema del emisor (a) y receptor (b) FHSS empleando secuencias ZC para el $k$-ésimo transductor.

\subsection{MODULACIÓN OFDM}

La modulación OFDM es una técnica de acceso al medio multiportadora que distribuye los datos en $N_{F F T}$ canales de frecuencia equiespaciados, en el ancho de banda de 0 a $\frac{f_{s}}{2} H z$, siendo $f_{s}$ la frecuencia de muestreo. El uso de subportadoras ortogonales admite el solapamiento, mejorando el uso del ancho de banda y aumentando la eficiencia espectral en comparación con otros esquemas de modulación multiportadora [9]. Este esquema de modulación puede ser implementado eficientemente usando la transformada de Fourier inversa (IFFT) en el emisor y la FFT en el receptor. La modulación multitono discreta (DMT) [4] es equivalente a OFDM, pero en su lugar, ésta utiliza una IFFT con el doble de muestras, es decir, $N_{F F T}=2 N$, aprovechando la propiedad de simetría Hermítica para emitir una señal real en banda base. Esta modulación puede expresarse como:

$T_{k}[l]=\sum_{n=0}^{2 N-1} S_{k}[n] \cdot e^{j 2 \pi n l / 2 N} ; \quad l=0, \ldots, 2 N-1$

donde $S_{k}[2 N-n]=S_{k}[n]^{*}$ y $S_{k}[n]^{*}$ es el complejo conjugado de $S_{k}[n]$, que identifica el símbolo del $k$-ésimo transductor , transmitido en la subbanda $n$, y $N$ es el número de canales ortogonales o por- 
tadoras. Teniendo en cuenta la segmentación del ancho de banda en DMT, para ubicar el código ZC en el ancho de banda apropiado del transductor, cada bit del código debe introducirse en los canales de la IFFT que correspondan a las frecuencias de salida deseadas, y el resto de las entradas deberán completarse con ceros. El número de ceros a añadir en la parte inferior y superior son $Z_{L}$ y $Z_{H}$ respectivamente, y dependen de la frecuencia de muestreo $f_{s}$, del ancho de banda total disponible $A B=f_{2}-f_{1}$, y de la longitud de la secuencia, como se expresa en (7).

$$
\begin{aligned}
Z_{H} & =\frac{L_{Z C}\left(f_{s}-2 f_{2}\right)}{2\left(f_{2}-f_{1}\right)} \\
Z_{L} & =\frac{2 f_{1}\left(L_{Z C}+Z_{H}\right)}{f_{s}-2 f_{1}}
\end{aligned}
$$

Los datos de entrada $S_{k}[n]$ en (6), antes de aplicarle la simetría Hermítica serán iguales a un vector de longitud $N$ :

$$
\begin{aligned}
S_{k}[n]= & {\left[0, \operatorname{ceros}\left(1: Z_{L}\right), Z_{r_{k}}[0], \ldots\right.} \\
& \left., Z_{r_{k}}\left[L_{Z C}-1\right], \operatorname{ceros}\left(1: Z_{H}\right)\right]
\end{aligned}
$$

La figura 6 muestra los esquemas del emisor y receptor. En el receptor, la señal entrante es directamente correlada con la señal modulada $T_{k}[l]$.

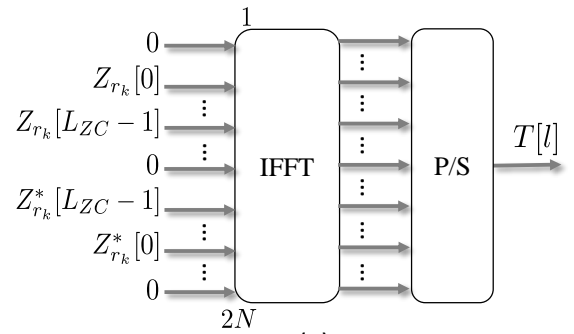

(a)

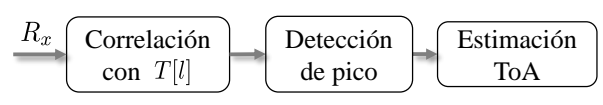

(b)

Figura 6: Esquemas del emisor (a) y receptor (b) OFDM, utilizando secuencias ZC para el $k$-ésimo transductor.

\section{RESULTADOS DE SIMULACIÓN}

En esta sección se presentan los resultados obtenidos con el sistema propuesto. Los esquemas de modulación se evaluaron en simulación utilizando MATLAB ${ }^{\circledR}$. Se ha considerado el posicionamiento local en ambientes interiores, añadiendo efectos adversos a las señales moduladas tales como: multicamino, efecto cerca-lejos, ruido blanco Gaussiano, ruido impulsivo y efecto Doppler.
Las señales fueron diseñadas en cuanto a longitud y número de saltos en frecuencia (para el caso FHSS), considerando un U-LPS formado por 5 transductores, con un ancho de banda aproximado de $B W=10 \mathrm{kHz}$ centrado en $40 \mathrm{kHz}$. La frecuencia de muestreo para la emisión es $f_{s}=500 \mathrm{kHz}$. Para una comparación justa, las señales se diseñaron aproximadamente con la misma duración. Con estas condiciones, en el caso de FHSS, sería deseable una duración de símbolo de 500 muestras (1 ms) para evitar el solapamiento entre portadoras. Sin embargo, se redujo a 350 muestras para disminuir la duración de la señal a $24 \mathrm{~ms}$, lo que por otro lado redunda en un menor efecto Doppler.

Las señales evaluadas son:

- Modulación QPSK: $L_{Z C}=257, N_{c}=4$ símbolos, $O_{f}=12$, tiempo de emisión $t_{e}=$ $24,67 \mathrm{~ms}$.

- Modulación OFDM: $L_{Z C}=191, Z_{L}=883$ y $Z_{H}=4894$ ceros, tiempo de emisión $t_{e}=$ $23,87 \mathrm{~ms}$.

- Modulación Slow-FH: $L_{Z C}=7$, longitud del símbolo 350 muestras, $k=5$ símbolos, tiempo de emisión $t_{e}=24,5 \mathrm{~ms}$. Un salto por cada cinco símbolos.

- Modulación Fast-FH: $L_{Z C}=7$, longitud del símbolo 350 muestras, $k=5$ símbolos, tiempo de emisión $t_{e}=24,5 \mathrm{~ms}$. Un salto por cada símbolo.

La precisión de los resultados se midió como la raiz del error cuadrático médio (RMSE, root mean square error) en muestras de la estimación del ToA.

\subsection{EFECTO MULTICAMINO}

En entornos interiores, un receptor detecta múltiples réplicas retardadas y atenuadas de una señal transmitida debido a reflexiones especulares en paredes y objetos. Por lo tanto, la señal recibida $R_{x}[n]$ puede ser modelada como:

$$
R_{x}[n]=\left(S[n]+\sum_{i=1}^{N_{M P}} \alpha_{i} S\left[n-d_{i}\right]\right)
$$

donde $N_{M P}$ representa el número de copias de la señal emitida $S[n]$; cada copia es retardada por $d_{i}$ muestras aleatorias y atenuada por una función exponencial con el parámetro $\alpha_{i}$, como puede verse en la figura 7 .

La figura 8 muestra el error absoluto acumulativo en la detección del pico de correlación sobre 500 iteraciones por esquema de modulación. Se seleccionó un número aleatorio de muestras de retardo 


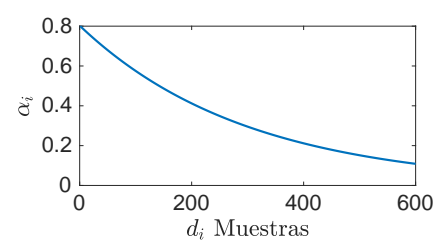

Figura 7: Factor de atenuación exponencial $\alpha_{i}$ como función del retardo en muestras aleatoriamente elegido.

por cada iteración para los $N_{M P}=6$ multicaminos. En esta figura, se puede observar que para QPSK, el $90 \%$ de las estimaciones tienen un error de menos de 5 muestras, mientras que el error para los otros tres esquemas en el $90 \%$ de la CDF es de 12 muestras o más, siendo OFDM el peor esquema alcanzando casi las 40 muestras.

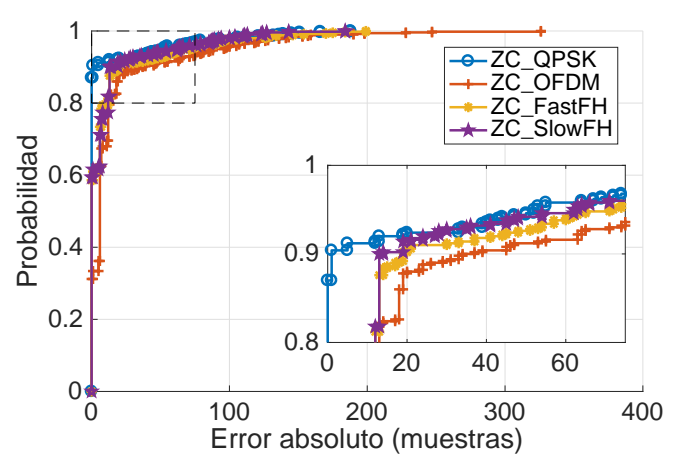

Figura 8: Error absoluto acumulativo en la detección del ToA, para una única emisión afectada por 6 multicaminos.

\subsection{EFECTO CERCA-LEJOS}

Cuando el receptor demodula una señal que es mucho más débil que las señales recibidas de las otras balizas, los picos de correlación cruzada debidos a esas otras señales pueden llegar a ser significativos. Como resultado, el detector puede ser incapaz de resolver la señal débil.

Para simular este efecto, se generaron dos secuencias ZC $\left(S_{1}\right.$ y $\left.S_{2}\right)$. La señal débil $\left(S_{2}\right)$ fue multiplicada por un factor de atenuación $\alpha$, retardada $d_{12}$ muestras, y sumada a la otra señal $\left(S_{1}\right)$ :

$$
R_{x}[n]=\left(S_{1}[n]+\alpha S_{2}\left[n-d_{12}\right]\right)
$$

Se calculó el error de ToA a la salida del correlador $S_{2}$ por cada valor de $\alpha$, separando cada secuencia $d_{12}=200$ muestras. La figura 9 muestra que, en un entorno libre de ruido, el pico de correlación es detectado correctamente utilizando QPSK incluso cuando la señal "lejana" de interés es atenuada al $14 \%$ de la señal "cercana". Los otros esquemas de modulación requieren una señal "lejana" más fuerte para una estimación correcta del ToA, al menos del $25 \%$ de la señal "cercana" en el caso de FHSS y mayor para OFDM. A medida que la intensidad de la señal "lejana" disminuye, también lo hace la amplitud del pico de correlación, produciendo que eventualmente este se vea superado por la correlación cruzada de la señal "cercana". En un caso libre de ruido, esto conduce a un salto repentino en la RMSE ya que superado ese "umbral" el retardo correspondiente al pico de correlación cruzada de la señal "cercana" que se identifica erróneamente suele ser el mismo.

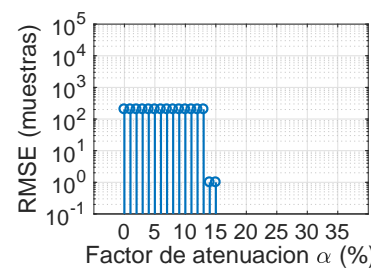

(a)

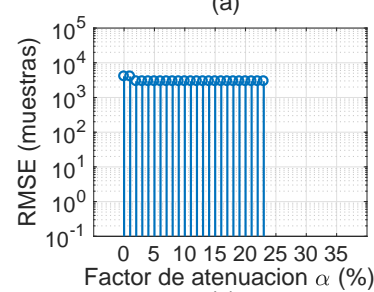

(c)

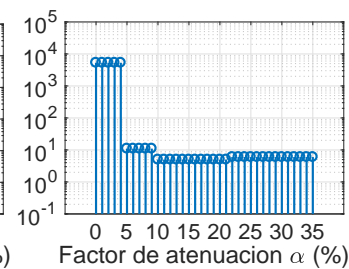

(b)

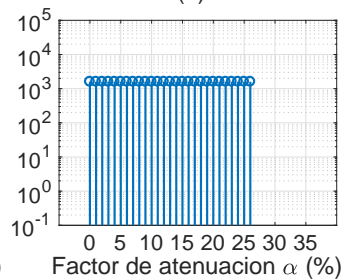

(d)
Figura 9: RMSE de la estimación del ToA con efecto cerca-lejos para los esquemas propuestos: (a) modulación QPSK, (b) OFDM, (c) Fast-FH y (d) Slow-FH).

\subsection{RUIDO BLANCO GAUSSIANO}

El ruido ambiental está siempre presente, creado por personas o máquinas, y puede afectar la estimación del ToA dependiendo de su energía y frecuencia. Para probar este comportamiento en los esquemas propuestos, se agregó ruido blanco Gaussiano a una única señal emitida variando la relación señal a ruido $(\mathrm{SNR})$ de $0 \mathrm{a}-35 \mathrm{~dB}$.

La figura 10 muestra el resultado del RMSE obtenido en la detección del ToA, a partir de un conjunto de 500 iteraciones por valor de SNR. En esta, se representan los resultados obtenidos sin aplicar el efecto de filtrado del transductor (a), y en (b) aplicando un filtro según se mencionó en la sección 3 , con un $B W=10 \mathrm{kHz}$ centrado en $40 \mathrm{kHz}$. Cuando no se aplican los efectos del transductor se observa que QPSK es el esquema más robusto al ruido en comparación con los demás, alcanzando niveles de SNR de hasta $-22 \mathrm{~dB}$ antes de obtener un RMSE de más de una muestra en la estimación del ToA. Por debajo de un SNR de $-25 \mathrm{~dB}$, todos los esquemas evaluados muestran RMSE simila- 
res. Sin embargo, cuando se agregan los efectos del transductor, en la figura 10 (b), se puede observar que el esquema QPSK empeora ascendiendo el valor de SNR a $-12 \mathrm{~dB}$ para RMSE menores a una muestra. Este deterioro se debe a que el espectro de frecuencia de la modulación QPSK no se encuentra tan acotado a $B W=10 \mathrm{kHz}$, y por ende parte de su energía se pierde luego de aplicar el filtrado de la señal, disminuyendo también la amplitud del pico de correlación.

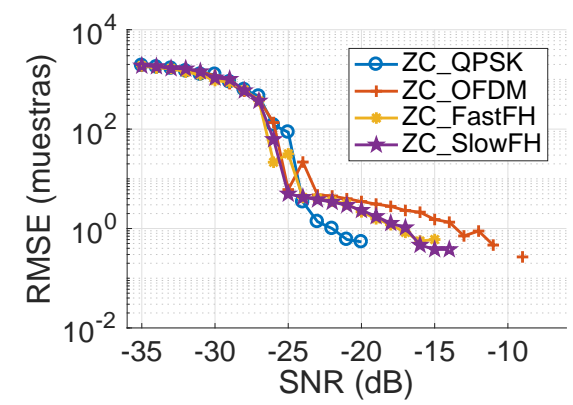

(a)

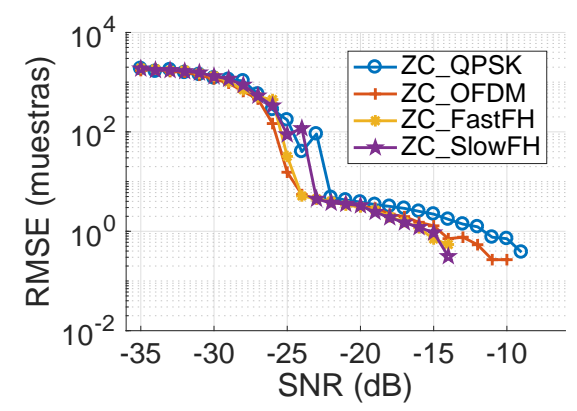

(b)

Figura 10: RMSE de la estimación del ToA al agregar ruido blanco Gaussiano a una única secuencia. En (a) las señales son afectadas por el ruido, mientras que en (b) además son filtradas a un $B W=10 \mathrm{kHz}$ centrado en $40 \mathrm{kHz}$.

\subsection{RUIDO IMPULSIVO}

La energía acústica producida por muchos fenómenos mecánicos contiene ruido audible y ultrasónico que puede causar un mal funcionamiento de los sistemas basados en ultrasonidos. Añadimos ruido Gaussiano blanco de banda ancha con un $S N R=-30 \mathrm{~dB}$ durante varios períodos de tiempo, afectando desde 0 al $95 \%$ de la duración de la señal emitida. Una única secuencia fue transmitida, para evitar la interferencia por correlación cruzada (CC). Los valores de RMSE se muestran en la figura 11, calculados a partir de 500 iteraciones por incremento porcentual, donde se puede observar que para duraciones menores al $30 \%$ de la longitud de señal, la mayoría de los esquemas presentan RMSE inferiores a 7 muestras, pero superado este valor, los errores en la estimación del
ToA comienzan a crecer abruptamente.

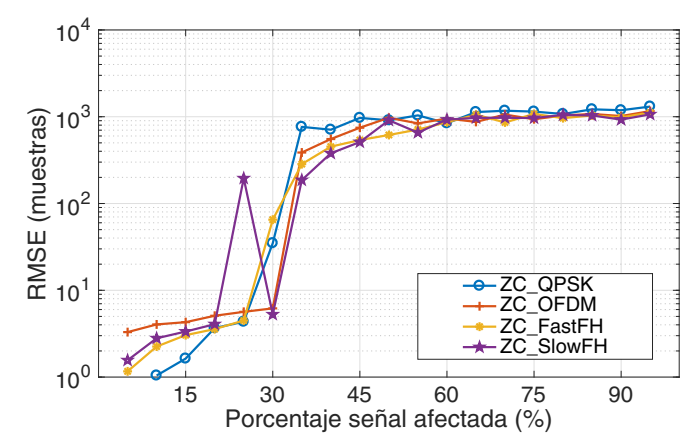

Figura 11: RMSE de la estimación del ToA de una única secuencia emitida afectada por ruido impulsivo a $\mathrm{SNR}=-30 \mathrm{~dB}$.

\subsection{EFECTO DOPPLER}

El movimiento del receptor produce un ensanchamiento o compresión de la señal emitida a medida que se aleja o se acerca al emisor, este efecto es modelado asumiendo una frecuencia de muestreo virtual $f_{s}^{\prime}$ para la señal emitida [8], dada por:

$$
f_{s}^{\prime}=f_{s}\left[1-\frac{\overrightarrow{v_{r}}}{c} \frac{\overrightarrow{r_{e}}-\overrightarrow{r_{r}}}{\left|\overrightarrow{r_{e}}-\overrightarrow{r_{r}}\right|}\right]
$$

donde $f_{s}$ es la frecuencia de muestreo real, $c \simeq$ $343 \mathrm{~m} / \mathrm{s}$ es la velocidad del sonido en el aire a una temperatura de $20{ }^{\circ} \mathrm{C}$ y a una presión de $1 \mathrm{~atm}, \overrightarrow{r_{r}}$ y $\overrightarrow{r_{e}}$ representan los vectores de posición del receptor y emisor respectivamente, y $\overrightarrow{v_{r}}$ es el vector de velocidad del receptor. A partir de esta frecuencia virtual, y luego de un proceso de interpolación y diezmado, se obtiene la señal adquirida por el receptor a la frecuencia de muestreo real.

La figura 12 muestra el error de detección en función de la velocidad del receptor para varios valores de velocidad, desde estática $(0 \mathrm{~m} / \mathrm{s})$ hasta $4 \mathrm{~m} / \mathrm{s}$, con el receptor aproximándose al emisor en la misma dirección del vector velocidad.

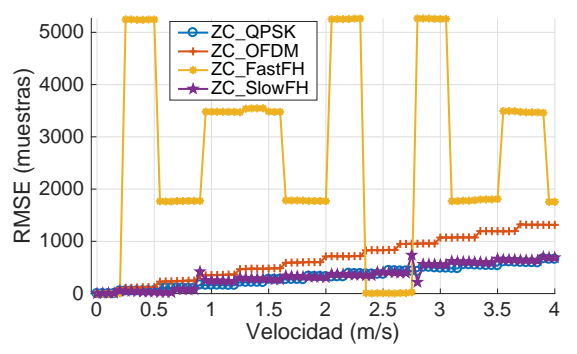

Figura 12: RMSE del error de detección con Doppler en función de la velocidad del receptor.

Las figuras 13 a 16 dan una visión más detallada de los efectos producidos en la función de $\mathrm{AC}$ 


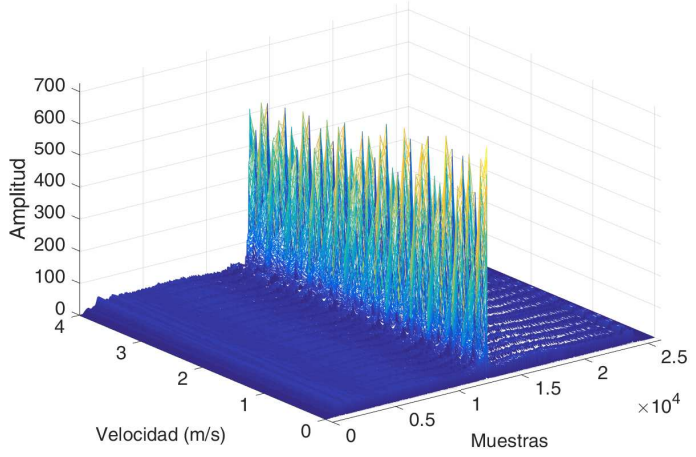

Figura 13: Vista del efecto Doppler en la correlación usando un esquema QPSK.

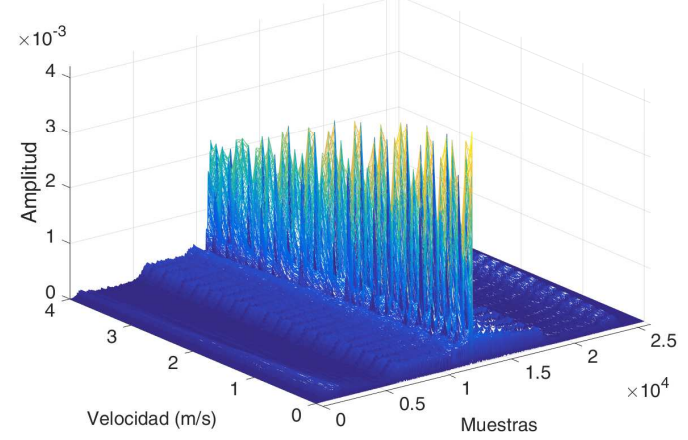

Figura 14: Vista del efecto Doppler en la correlación usando un esquema OFDM.

por el movimiento del receptor, para los cuatro esquemas de modulación. En ellos se puede observar que el peor esquema es Fast-FH debido al desvanecimiento de la amplitud del pico principal y la presencia de lóbulos laterales. En términos de robustez al Doppler, los mejores esquemas son los esquemas QPSK y OFDM. En ambos casos, la amplitud se degrada ligeramente y el pico principal sufre una pequeña desviación, que puede corregirse si se conoce la velocidad del receptor.

\section{CONCLUSIONES}

Se realizaron pruebas de simulación para evaluar las prestaciones de cuatro esquemas de modulacion codificados con secuencias Zadoff-Chu (QPSK, OFDM, Slow-FH y Fast-FH) en un sistema de posicionamiento local ultrasónico (U-LPS) en presencia de efectos adversos típicos de la señal en interiores tales como multicamino, efecto cercalejos, ruido impulsivo y desplazamiento Doppler. Se constató que la modulación QPSK proporciona la máxima precisión en términos de estimación de tiempo de llegada (ToA). Se debe llevar a cabo

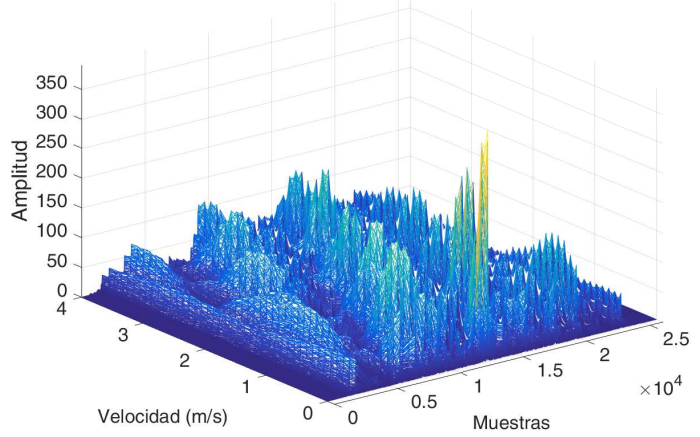

Figura 15: Vista del efecto Doppler en la correlación usando un esquema Fast-FH.

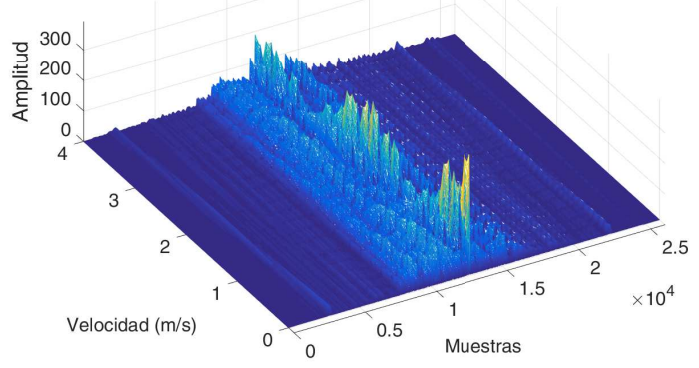

Figura 16: Vista del efecto Doppler en la correlación usando un esquema Slow-FH.

una investigación adicional realizando pruebas en entornos reales, para comparar y validar los resultados obtenidos.

\section{Agradecimientos}

Este trabajo ha sido posible gracias a la financiación del Ministerio Español de Economía y Competitividad (Proyecto SOC-PLC, ref. TEC201564835-C3-2-R), de la Universidad de Alcalá (CCGP2017-EXP/050) y la Universidad Nacional de la Patagonia San Juan Bosco de Argentina.

\section{English summary}

\section{DESIGN OF MODULATION SCHEMES IN A ZADOFF-CHU ENCODED ULTRASONIC POSI- TIONING SYSTEM}

\author{
Abstract \\ Many Ultrasonic Local Positioning Sys- \\ tems (U-LPS), consists of multiple fixed
}


beacons that simultaneously emit ultrasonic signals whose times of arrival (ToA) are estimated at a mobile receiver. The ultrasonic signals can be encoded to allow separation of the signals at the receiver, identification of the transmitters, and mitigation of signal impairments such as multipath, the near-far effect, multiple access interference, and Doppler shift. Therefore, waveform design (coding and modulation) constitutes a key factor in determining the performance of such a system. This paper compares by simulation the performance of three modulation techniques (specifically QPSK, OFDM and FHSS) transmitting Zadoff-Chu (ZC) sequences in an U-LPS. Simulation results indicate that $Z C$ coded QPSK modulation with non-coherent detection, offers greater accuracy under realistic conditions than the other modulation schemes.

Keywords: Zadoff-Chu sequences, QPSK, OFDM, frequency hopping, ultrasound, indoor positioning.

\section{Referencias}

[1] AlSharif, M. H., Saad, M.,Siala, M., Ballal, T., Boujemaa, H., and Al-Naouri, T. Y. (2017) "Zadoff-chu coded ultrasonic signal for accurate range estimation", 25th European Signal Processing Conference (EUSIPCO), pp. 1250-1254.

[2] Chu, D. (1972) "Polyphase codes with good periodic correlation properties (Corresp.)", IEEE Transactions on Information Theory, vol. 18 , pp. 531-532.

[3] Frank, R., Zadoff, S., and Heimiller,R. (1962) "Phase shift pulse codes with good periodic correlation properties (Corresp.)", IRE Transactions on Information Theory, vol. 8, pp. 381-382.

[4] García, E., Paredes, J. A., Álvarez, F. J., Pérez, M. C., and García, J. J. (2015) "Spreading sequences in active sensing:A review", Signal Processing, vol. 106, pp. 88-105.

[5] García, E., Ureña, J., Gualda, D., Hernández, A., and Nombela, F. (2015) "Discrete Multitone Modulation for Ultrasonic Indoor Positioning Systems", International Conference on Indoor Positioning and Indoor Navigation (IPIN).

[6] Gonzalez, J. R. and Bleakley, C. J. (2009) "High-precision robust broadband ultrasonic location and orientation estimation", IEEE Journal of Selected Topics in Signal Processing, vol. 3, pp. 832-844.

[7] Kasami, T. (1966) "Weight distribution formula for some class of cyclic codes", Technical Report AD0632574, Coordinated Science Lab. University of Illinois.

[8] Paredes, J. A., Aguilera, T., Álvarez, F. J., Lozano, J., and Morera, J. (2011) "Analysis of doppler effect on the pulse compression of diferent codes emitted by an ultrasonic LPS", Sensors, vol. 11, pp. 10765-10784.

[9] Stanciu, A. E., Nemţoi, L.-M., and Moise, I. M. (2012) "Considerations regarding the spectral efficiency of orthogonal frequency division multiplexing", International Conference on Development and Application Systems.

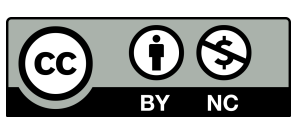

C) 2018 by the authors. Submitted for possible open access publication under the terms and conditions of the Creative Commons Attribution CC-BY-NC 3.0 license (http://creativecommons.org/licenses/by-nc/3.0/). 